\title{
Study Day of Respiratory System Findings
}

National Cancer Institute

\section{Source}

National Cancer Institute. Study Day of Respiratory System Findings. NCI Thesaurus.

Code C123952.

The study day that a respiratory system assessment is performed. 